\title{
Research on Dynamic Reliability of a Jet Pipe Servo Valve Based on Generalized Stochastic Petri Nets
}

\author{
Yuanbo Chu, Zhaohui Yuan, and Jia Chen \\ School of Automation, Northwestern Polytechnical University, Xian, Shaanxi 710072, China \\ Correspondence should be addressed to Yuanbo Chu; chuyuanbo528@163.com
}

Received 3 May 2015; Revised 23 June 2015; Accepted 28 June 2015

Academic Editor: Hui Hu

Copyright (C) 2015 Yuanbo Chu et al. This is an open access article distributed under the Creative Commons Attribution License, which permits unrestricted use, distribution, and reproduction in any medium, provided the original work is properly cited.

\begin{abstract}
The jet pipe servo valve is widely used in the military fields of aviation and ship, whose reliability has obvious randomness and dynamic. However, existing methods are either having complicated theory or analyzing static reliability. Based on the generalized stochastic petri nets (GSPN) theory and the collected basic failure modes and failure rate data of jet pipe servo valve, this paper proposes a novel modeling and simulating method for system's dynamic behavior analysis. In this method, the dynamic reliability model considering failure's random and repair is established and is simulated using GSPN software. Then, the steady state probability of servo valve is calculated, which is compared with the value calculated by Markov method. Finally, the dynamic reliability parameters of jet pipe servo valve are calculated using collected failure rate data and different repair rate data. Results show the probability that the maximum error between methods of GSPN and Markov is $2.07 \%$, the optimal repair rate set is less than $1.71 \mu_{i}$, and also the dynamic reliability parameters become better with increasing simulation time because of failure's recovery. Therefore, research methods and results based on GSPN are concise and realistic, which can be used for failure's qualitative forecast and dynamic reliability's quantitative calculation of similar complicated system.
\end{abstract}

\section{Introduction}

The operational requirements such as all-weather, rapid response, and precision strike demand higher performance and function of military equipment. The internal features such as complex structure and high density request higher reliability, lifetime, and environmental adaptability of military equipment. Moreover, the design parameters (such as size, strength), the environments (such as temperature, stress, and road conditions), and the failure's process of military equipment have obvious randomness and dynamic. All the above propose new challenge for traditional methods of equipment design and reliability analysis. In these dynamic and competitive environments, military manufacturing enterprises are facing increasingly fierce market pressure. They are required not only to reduce costs and shorten the production cycle but also to improve product's quality, enhance the competitiveness, and ensure sustainable development of military enterprises, which urgently require people to build dynamic reliability theory of large and complex systems [1-3].
Unlike static reliability theory, the study object of dynamic reliability theory is a dynamic system. In fact, almost all engineering systems are dynamic systems. Dynamic system can be defined as such a system, the response from which to initial disturbance changes with the interaction of system's components and the interaction of components and working environment, where the "dynamic" deliberately emphasizes the time course of events and the importance of the interaction among system's components $[4,5]$. The dynamic reliability theory is mainly used to analyze the behavior of failure's randomness and repair, the dynamic characteristics, and the interaction between operators and work environment. From the existing literature, the studies of most dynamic reliability models are for complex systems; the modeling theories of dynamic reliability mainly consist of state transition diagram [6], go-flow [7], dynamic fault tree [8], stochastic petri nets [9], and Monte Carlo simulation method [10]. Among them, stochastic petri nets method is the most widely used method in the dynamic reliability research because of its solid theoretical foundation, graphical modeling, and easy operational software. However, 
the stochastic petri nets method has state explosion problem in reliability modeling for large and complex systems; therefore, the generalized stochastic petri nets (GSPN) method has been proposed [11].

Electrohydraulic servo valve is an important bridge to connect the electrical parts and hydraulic parts of electrohydraulic servo control system. Currently, in the military fields of aviation and ship, jet pipe servo valve has generally been used to replace the kind of nozzle flapper, whose reliability directly determines the reliability and safety of the military equipment's electrohydraulic servo system. Based on the actual working conditions and failure rate data of jet pipe servo valve, many scholars have done active researches about its reliability. Zhou et al. [12] collected failure modes within life stage of jet pipe servo valve and identified the weaknesses using the failure modes, effect, and criticality analysis (FMECA). Dai et al. [13] optimized FMECA for the jet pipe servo valve using the fuzzy evaluation method and gave the risk level of servo valve's each failure mode. Lyu et al. [14] combined the methods of Monte Carlo and fault tree to analyze reliability's digital simulation of jet pipe servo valve and got the mean time between failures (MTBF) and reliability under different simulation time. However, the growing fields of aviation and ship require the ability to quantitatively calculate the actual impact of failure's randomness and repair on servo valve system's reliability, that is, to quantitatively calculate the dynamic reliability of jet pipe servo valve.

Considering characters and working conditions of jet pipe servo valve, this paper presents a new method for assessing large and complex system's dynamic reliability. The remaining parts are organized as follows. Section 2 provides an overview of GSPN basic theory. Section 3 presents the new method for assessing system's dynamic reliability. Section 4 assesses jet pipe servo valve's dynamic reliability based on the method in Section 3. Section 5 gives the results and discussion. At last, the conclusion is given in Section 6 .

\section{Basic Theory of GSPN}

2.1. Definition of GSPN. Petri nets are extremely useful for reliability evaluation and description of systems characterized by sequentiality and concurrency. With the aim of extending the modelling power of stochastic petri nets, generalized stochastic petri nets were proposed [15].

Formally, a generalized stochastic petri nets model is an 8-tuple:

$$
\mathrm{GSPN}=\left\{P, T, \Pi, I, O, H, W, M_{0}\right\}
$$

where

(i) $P=\left\{p_{1}, p_{2}, \ldots, p_{n}\right\}$ is a finite set of places, which represents the state or resource of the system,

(ii) $T=\left\{t_{1}, t_{2}, \ldots, t_{n},\right\}$ is a finite set of transitions, which is the transition events changing state of the system:

$$
\begin{aligned}
T & =T_{\mathrm{im}} \cup T_{\text {tim }} ; \\
\mathrm{T}_{\mathrm{im}} \cap \mathrm{T}_{\mathrm{tim}} & =\emptyset,
\end{aligned}
$$

where $T_{\text {im }}$ means immediate transitions and $T_{\text {tim }}$ means timed transitions,

(iii) $\Pi: T \rightarrow N$ is the priority function that maps transitions onto natural numbers representing their priority levels:

$$
\begin{array}{ll}
\Pi\left(t_{k}\right)=0 ; & \forall t_{k} \in T_{\text {tim }}, \\
\Pi\left(t_{k}\right)>0 ; & \forall t_{k} \in T_{\mathrm{im}},
\end{array}
$$

(iv) $I, O, H: T \rightarrow \operatorname{Bag}(P)$ are the input, output, and inhibition functions, respectively. $\operatorname{Bag}(P)$ is the multiset on $P$,

(v) $W:(T \times M) \rightarrow R^{+}$is the stochastic function that maps transitions onto real numbers representing their firing rates depending on the marking $M$,

$$
W=\left(t_{k}, M\right)= \begin{cases}\lambda_{k}, & \text { if } t_{k} \in T_{\mathrm{tim}} \\ \omega_{k}, & \text { if } t_{k} \in T_{\mathrm{im}}\end{cases}
$$

(vi) $M_{0}: P \rightarrow N$ is the initial marking, a function that associates each place with a natural number.

Compared with SPN, GSPN has three improvements as follows [16]:

(1) Setting the immediate transition and timed transition, by which the logic of events and time consuming can be distinguished and the problem of state space explosion can be effectively alleviated. If the immediate transition and timed transition exist at the same time, only immediate transition is allowed to execute. The timed transition can be used to describe failure's randomness and repairing process, so that the established model is more realistic.

(2) Increasing the inhibition arc. The inhibition arc is usually used to denote the failure's random and repair process while GSPN is used to analyze system's reliability; when the number of tokens is equal to the number of the inhibition arcs, the transition is inhibited to execute.

(3) Defining random switch, if several immediate transitions are enabled at the same time, whose priorities can be defined. If their priorities are the same, the execution probability of the immediate transition is determined by the random switch.

2.2. Graphical Modeling Elements of GSPN. The theory of GSPN is used for dynamic reliability's modeling, simulating, and parameter's calculating of jet pipe servo valve, and the meaning of symbols during modeling and simulating is shown as Table 1.

\section{The Analytical Methods of GSPN Model}

GSPN can be applied to assess the dynamic reliability of large and complex system, such as calculating the reliability 
TABLE 1: The meaning of symbols during reliability modeling and simulating.

\begin{tabular}{lcc}
\hline Symbols & Graph & Meaning \\
\hline$p$ & & $\begin{array}{c}\text { The state of system or } \\
\text { subsystem } \\
\text { Immediate transition } \\
t_{\text {im }}\end{array}$ \\
$t_{\text {tim }}$ & Timed transition \\
$k$ & $\longrightarrow$ & Mark of system or \\
$v$ & & subsystem \\
$f$ & $\longrightarrow$ & Allowing arc \\
\hline
\end{tabular}

parameters, optimizing the repair rate data, and forecasting the failures of the dynamic system. The procedure of analytical methods based on GSPN presented in this paper is put forward as follows.

Step 1 (system's mechanism analysis). System's mechanism analysis is the foundation of dynamic reliability assessment, which consists of principle analysis, structure decomposition, and failure mode's summary.

Step 2 (data collection of failure rate and repair rate). The aim of dynamic reliability's quantitative analysis is to forecast dynamic system's reliability parameters based on subsystem's failure rate data and repair rate data. There are several sources to find this information such as public data books, performance data from the actual plant, expert judgments, and laboratory testing [6]. In this step, we also need to define each subsystem and to set the data of failure rate and repair rate using GSPN software.

Step 3 (dynamic reliability model's establishing based on GSPN). Based on previous two steps, the GSPN model of the system can be established. It is worth mentioning that the failure time and repair time of the subsystems are not required to obey the exponential distribution in the GSPN model, but if the failure time and repair time of all subsystems obey exponential distribution, the GSPN model has the same structure as a Markov model.

Step 4 (simulation and method validation). When finishing the graph's connection and setting data, we can run simulation experiments. Moreover, if all subsystem's failure time and repair time obey exponential distribution, we can compare the result calculated by GSPN method with Markov method to verify the validity.

Step 5 (repair rate's optimization). All subsystem's failure rate and repair rate have a significant impact on dynamic reliability parameters of the system. The failure rate mainly depends on the statistical data. However, the repair rate depends on the repair strategy, which consists of timely repair, regular overhaul, and reliability centered maintenance (RCM); among them, the RCM is the core of modern maintenance theory. In this step, we should optimize subsystem's repair rate on the basis of reliability's satisfaction.
TABLE 2: The failure rate data and repair rate data of the key elements.

\begin{tabular}{lcc}
\hline Subsystem & $\begin{array}{c}\text { Failure distribution } \\
\lambda_{i}\left(10^{-6} / \mathrm{h}\right)\end{array}$ & $\begin{array}{c}\text { Repair } \\
\text { distribution } \\
\mu_{i}\left(10^{-3} / \mathrm{h}\right)\end{array}$ \\
\hline Spool $\left(p_{1}\right)$ & Exponent, 0.1 & Exponent, 0.5 \\
Valve body $\left(p_{2}\right)$ & Exponent, 0.1 & Exponent, 0.5 \\
Bush $\left(p_{3}\right)$ & Exponent, 0.1 & Exponent, 0.5 \\
Jet pipe $\left(p_{4}\right)$ & Exponent, 2.0 & Exponent, 1.0 \\
Nozzle $\left(p_{5}\right)$ & Exponent, 2.0 & Exponent, 1.0 \\
Receiver $\left(p_{6}\right)$ & Exponent, 2.0 & Exponent, 1.0 \\
Permanent magnet $\left(p_{7}\right)$ & Exponent, 0.2 & Exponent, 1.0 \\
Armature $\left(p_{8}\right)$ & Exponent, 0.2 & Exponent, 1.0 \\
Guide magnet $\left(p_{9}\right)$ & Exponent, 0.2 & Exponent, 1.0 \\
Spring pipe $\left(p_{10}\right)$ & Exponent, 5.0 & Exponent, 1.0 \\
Control coil $\left(p_{11}\right)$ & Exponent, 3.3 & Exponent, 1.0 \\
\hline
\end{tabular}

\section{GSPN Model of Jet Pipe Servo Valve}

Step 1 (system's mechanism analysis). Electrohydraulic servo valve is a kind of precision hydraulic components capable of proportionally converting weak electrical control signal into large hydraulic power and outputting a corresponding flow and pressure, which mainly consists of slide valve components, jet pipe amplifier, permanent magnet torque motor, and feedback components. Among them, the slide valve components are composed of the spool, bush, and valve body; the jet pipe amplifier consists of the jet pipe, nozzle, and receiver; the permanent magnet torque motor consists of permanent magnets, guide magnet, armature, spring pipe, and control coils (dual redundant mode); the feedback components are composed of feedback elements and clamping screws. The structural decomposition block diagram of jet pipe servo valve is shown as Figure 1 and the failure modes are shown in reference of Zhou and Dai et al. $[12,13]$.

Step 2 (data collection of failure rate and repair rate). On the basis of structure and failure mode, the key structure elements affecting reliability of jet pipe servo valve are determined. Through searching literature and estimate by Lyu et al. [14], the failure rate data and repair rate data of the key elements are shown as Table 2.

Step 3 (dynamic reliability model's establishing based on GSPN). Consider the following.

(a) Reliability Block Diagram. On the basis of servo valve's key structure elements, working principle, and the failure modes, the reliability block diagram of servo valve is shown as Figure 2.

(b) Modeling Premise. When establishing servo valve's dynamic reliability model based on GSPN, it is necessary to assume three points as follows: 


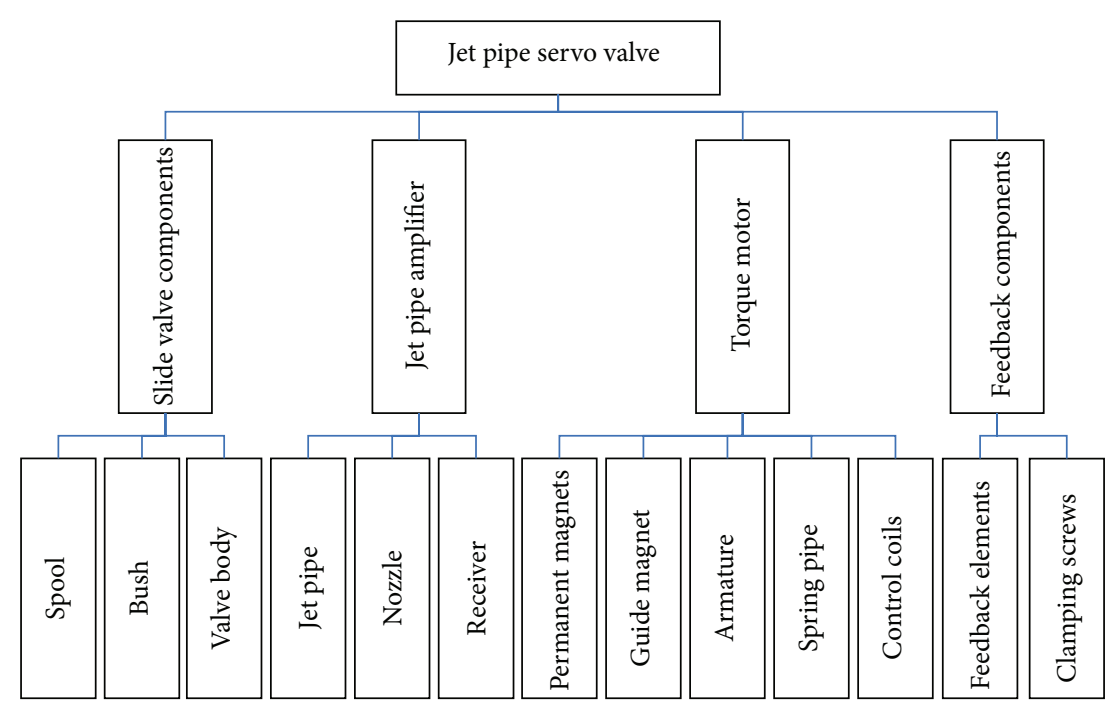

FIgURE 1: The structural decomposition block diagram of jet pipe servo valve.

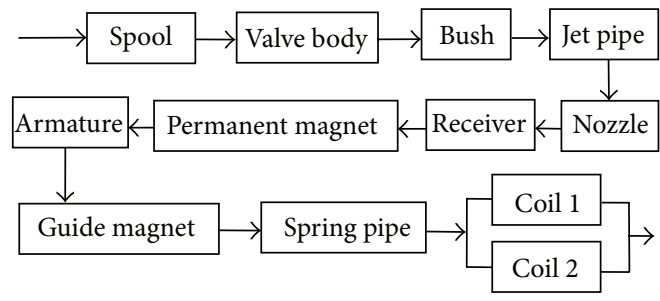

FIGURE 2: The reliability block diagram of servo valve.

(1) Servo valve system and subsystem have only two states: normal or failure.

(2) Failures are repaired immediately after taking place.

(3) Hydraulic components are repaired as new after failure.

(c) GSPN Model of Servo Valve's Dynamic Reliability. Based on reliability block diagram and subsystem's failure distribution, the GSPN model of jet pipe servo valve's dynamic reliability is established as Figure 3. In Figure 3, $p_{i} . o(i=1,2, \ldots, 11)$ and $p_{i} \cdot n(i=1,2, \ldots, 11)$, respectively, denote that the subsystem is in normal and failure states, $p_{s .} o$ and $p_{s} . n$, respectively, represent that the system is in normal and failure states, and $t_{2 n-1}(n=1,2, \ldots, 11)$ and $t_{2 n}(n=1,2, \ldots, 11)$ are timed transitions, which, respectively, denote the excitation of subsystem's failure and repair. Take the subsystem of spool as an example to explain servo valve's failure and repair process. At the beginning of the system's running, $t_{25}$ are enabled and execute; its result is $p_{s .} o$ and $p_{i .} . o(i=1,2, \ldots, 11)$ receive tokens, which indicates servo valve is in normal working state. After a random normal working time $t_{1}$, the subsystem of spool fails to work and $p_{1} . n$ receive token, which indicates that subsystem of spool is in the state of failure. At this time, $t_{27}$ is enabled and executes, $p_{s} . n$ receive token, and servo valve is in the state of failure; then, $t_{26}$ executes and the tokens of $p_{s .} . o$ and $p_{s .} n$ are removed, which leads to the execution again of $t_{27}$, and $p_{s} n$ receive token, when other subsystems are forbidden to fail because of the existence of prohibiting arcs. Then, the subsystem of spool begins to be repaired. After a random repair time $t_{2}, t_{25}$ executes and finally leads to $t_{27}$ 's enablement and execution. After that, the tokens of $p_{s .} .0$ and $p_{s} . n$ are removed at the same time; the system of servo valve goes back to normal working state again.

Step 4 (simulation and method validation). Consider the following.

(a) Simulation. Setting up the simulation time and related parameters, the dynamic reliability simulation experiments are carried out and the experimental data is collected.

(b) Method Validation. In order to verify the GSPN method's effectiveness in the dynamic reliability analysis of servo valve, the Markov method is introduced, whose state transition diagram is shown as Figure 4. The servo valve system has only two working states; the first is that subsystems are all in normal working state and the servo valve system is in normal state; the other is that only one of the eleven subsystems fails to work; then, the entire system is in failure state.

In Figure 4 , the states $M_{1}, M_{2}, \ldots, M_{11}$ cannot be directly transferred to each other; the state transition probability matrix $P$ and density matrix $A$ of the servo valve system are shown as follows:

$$
P=\left[\begin{array}{ccccc}
1-\sum_{i=1}^{11} \lambda_{i} & \lambda_{1} & \lambda_{2} & \cdots & \lambda_{11} \\
\mu_{1} & 1-\mu_{1} & 0 & \cdots & 0 \\
\mu_{2} & 0 & 1-\mu_{2} & \cdots & 0 \\
\vdots & \vdots & \vdots & \ddots & \vdots \\
\mu_{11} & 0 & 0 & \cdots & 1-\mu_{11}
\end{array}\right],
$$




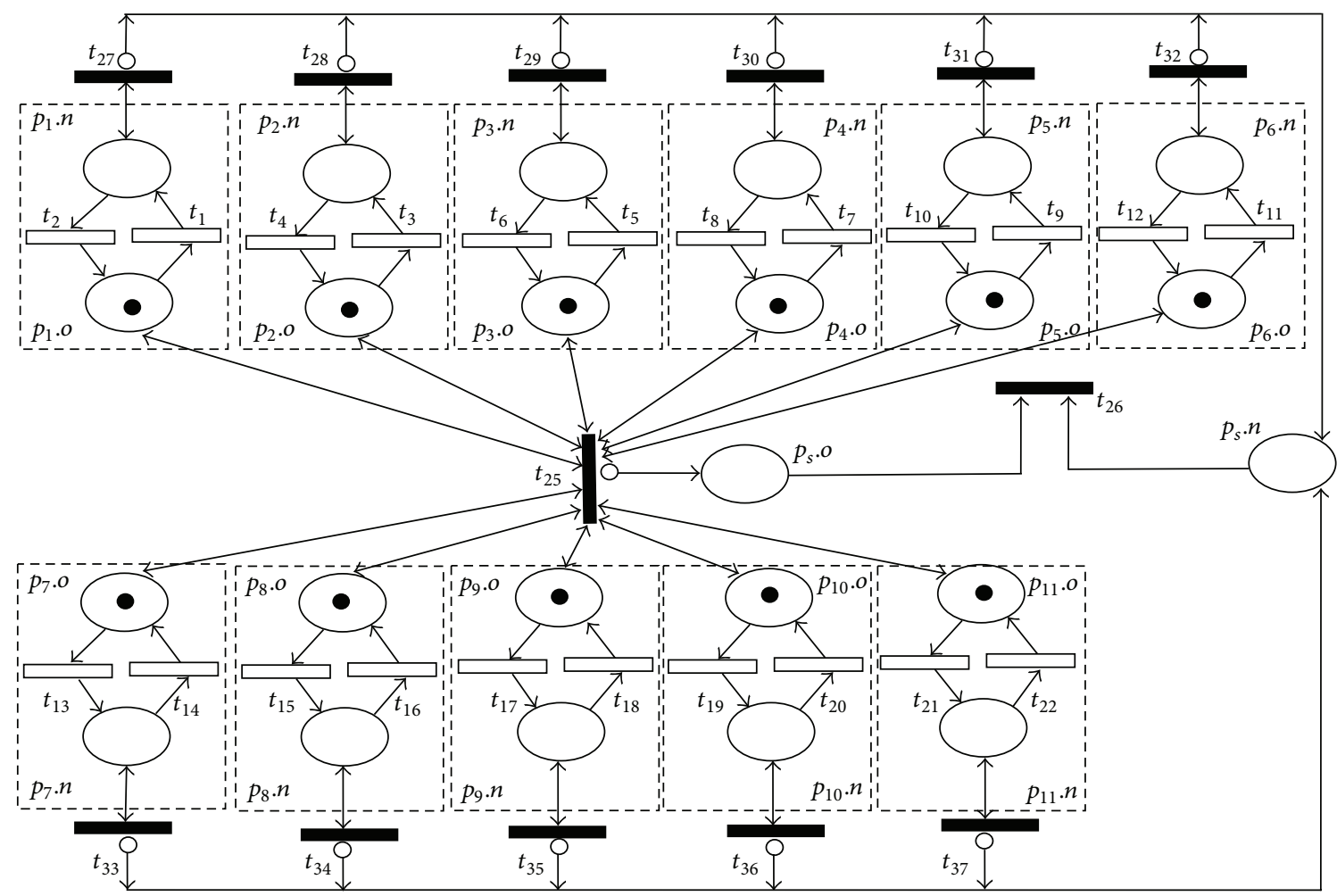

FIGURE 3: The GSPN model of jet pipe servo valve's dynamic reliability.

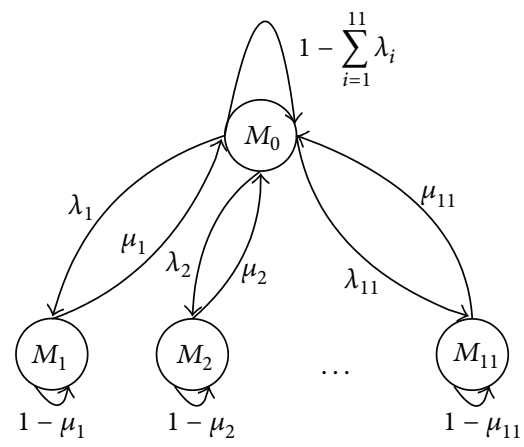

Figure 4: The Markov state transition diagram of servo valve.

$$
A=\left[\begin{array}{ccccc}
-\sum_{i=1}^{11} \lambda_{i} & \lambda_{1} & \lambda_{2} & \cdots & \lambda_{11} \\
\mu_{1} & -\mu_{1} & 0 & \cdots & 0 \\
\mu_{2} & 0 & -\mu_{2} & \cdots & 0 \\
\vdots & \vdots & \vdots & \ddots & \vdots \\
\mu_{11} & 0 & 0 & \cdots & -\mu_{11}
\end{array}\right],
$$

where $\lambda_{i}$ and $\mu_{i}$ are, respectively, the failure rate and repair rate of subsystems.

When transition probability matrix and density matrix are determined, servo valve system's steady state probability can be got by solving (6), and the normal working state and failure state's probability are calculated as (7) and (8):

$$
\begin{aligned}
P A & =0 \\
\sum_{i=0}^{11} P_{i} & =1, \\
P_{M 0} & =\left(1+\sum_{i=1}^{11} \frac{\lambda_{i}}{\mu_{i}}\right)^{-1}, \\
P_{M i} & =\frac{\lambda_{i}}{\mu_{i}}\left(1+\sum_{i=1}^{11} \frac{\lambda_{i}}{\mu_{i}}\right)^{-1} .
\end{aligned}
$$

The simulation time is set as 50000 hours; then, the failure rate data and repair rate data of all subsystems are set according to Table 2; the steady state probability of servo valve system's working state $\left(M_{0}, M_{1}, \ldots, M_{11}\right)$ is calculated, which is compared with the value calculated by Markov methods; the errors between them are shown as Table 3. From the error data of Table 3 , it is found that the maximum error is $2.07 \%$, which can be revised when the dynamic reliability parameters are calculated, so the GSPN method can be used to analyze the dynamic reliability of jet pipe servo valve.

Step 5 (repair rate's optimization and parameters' calculation). Consider the following.

(a) Repair Rate's Optimization. The repair rate data directly affects some parameters of dynamic reliability of servo valve 
TABLE 3: The errors of steady state probability between methods of Markov and GSPN.

\begin{tabular}{lccc}
\hline$M_{i}$ & Markov & GSPN & Error \\
\hline$M_{0}$ & 0.984737 & 0.985014 & $0.03 \%$ \\
$M_{1}$ & 0.000197 & 0.000193 & $2.07 \%$ \\
$M_{2}$ & 0.000197 & 0.000199 & $1.01 \%$ \\
$M_{3}$ & 0.000197 & 0.000197 & $0.00 \%$ \\
$M_{4}$ & 0.001969 & 0.001974 & $0.25 \%$ \\
$M_{5}$ & 0.001969 & 0.001968 & $0.05 \%$ \\
$M_{6}$ & 0.001969 & 0.001965 & $0.20 \%$ \\
$M_{7}$ & 0.000197 & 0.000199 & $1.01 \%$ \\
$M_{8}$ & 0.000197 & 0.000198 & $0.51 \%$ \\
$M_{9}$ & 0.000197 & 0.000201 & $1.99 \%$ \\
$M_{10}$ & 0.004924 & 0.004937 & $0.26 \%$ \\
$M_{11}$ & 0.003250 & 0.003265 & $0.46 \%$ \\
\hline
\end{tabular}

system. The failure rate data of the spool, the valve body, and the bush are all $0.1\left(10^{-6} / \mathrm{h}\right)$, whose repair rate data are all set as $0.5\left(10^{-3} / \mathrm{h}\right)$; other subsystems' repair rate data are all set as $1.0\left(10^{-3} / \mathrm{h}\right)$ based on the actual maintenance cycle and the failure rate data, so the data set of initial repair rate $\mu_{i}=(0.5,0.5,0.5,1.0,1.0,1.0,1.0,1.0,1.0,1.0,1.0)\left(10^{-3} / \mathrm{h}\right)$ for all subsystems is determined; the relationship of repair rate data and subsystem is shown as Table 2 . In the simulation experiments, $0.2 \mu_{i}, 0.4 \mu_{i}, 0.6 \mu_{i}, 0.8 \mu_{i}, 1.0 \mu_{i}, 1.2 \mu_{i}, 1.4 \mu_{i}, 1.6 \mu_{i}$, $1.8 \mu_{i}$, and $2.0 \mu_{i}$ are, respectively, set as repair rate data. According to (9), the availability of servo valve is calculated and is shown as Table 4 . In MATLAB, the above data is fitted as Figure 5, from which we can find the relationship among them is approximately linear. The relationship can be represented as (10):

$$
A=\frac{T_{w}}{T_{w}+T_{r}},
$$

where $T_{r}$ is the sum of all subsystems' repair time and $T_{w}$ is the working time of servo valve system. Consider

$$
A=-0.01718 M+0.9994,
$$

where $A$ is the dynamic availability of servo valve system and $M$ is the multiple of initial repair rate.

According to military equipment's strict requirement, if the dynamic availability of servo valve is higher than 0.95 when working time reach 50000 hours. Considering the maximum error $2.07 \%, M$ is less than 1.71 , calculated in accordance with (10).

(b) Dynamic Reliability Analysis of Servo Valve Based on GSPN. Considering certain safety margin, $M$ is set as 1 and the repair rate set is $\mu_{i}=(0.5,0.5,0.5,1.0,1.0,1.0,1.0,1.0,1.0$, $1.0,1.0),\left(10^{-3} / \mathrm{h}\right)$; then, the simulation time is, respectively, set as 1000 hours, 10000 hours, 100000 hours, 1000000 hours, and 10000000 hours; the simulation experiments are carried out to obtain servo valve's dynamic reliability parameters.
TABLE 4: The relationship between servo valve's availability and multiple of initial repair rate.

\begin{tabular}{lc}
\hline Multiple of initial repair rate & Availability $(A)$ \\
\hline 0.2 & 0.996 \\
0.4 & 0.993 \\
0.6 & 0.989 \\
0.8 & 0.985 \\
1.0 & 0.982 \\
1.2 & 0.978 \\
1.4 & 0.975 \\
1.6 & 0.972 \\
1.8 & 0.969 \\
2.0 & 0.965 \\
\hline
\end{tabular}

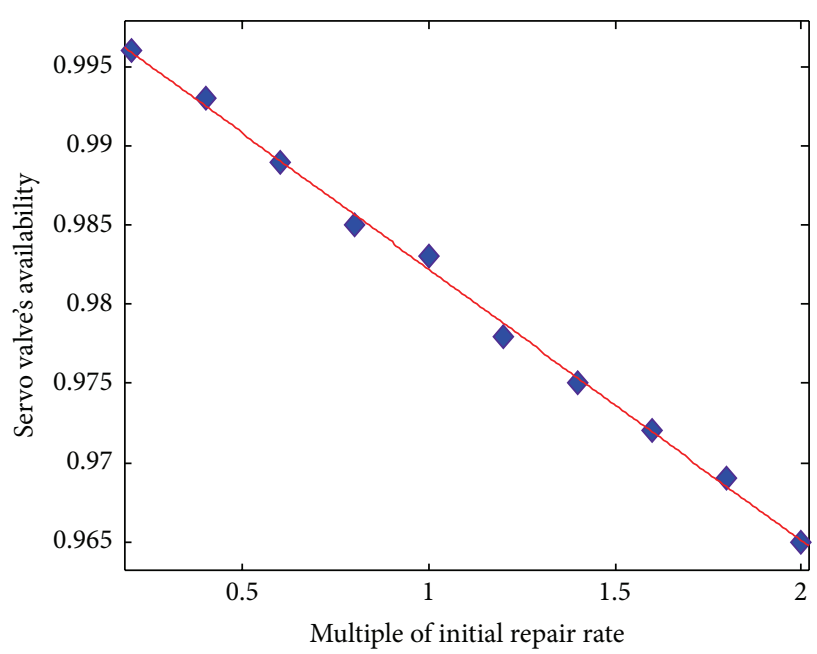

FIGURE 5: The relationship curve between servo valve's availability and multiple of initial repair rate.

Collecting simulation data and calculating through (11), the failure rate $(\lambda)$, availability $(A)$, mean time to restoration (MTTR), and meantime between failures (MTBF) are obtained and shown as Table 5. Consider

$$
\begin{aligned}
\lambda & =\frac{1}{\mathrm{MTBF}}, \\
\text { MTTR } & =\frac{T_{r}}{\sum_{i=1}^{11} m_{i}}, \\
\text { MTBF } & =\frac{T_{w}}{\sum_{i=1}^{11} m_{i}},
\end{aligned}
$$

where $m$ is the failure times of the subsystems.

Using the Curve Fitting Tool, the dynamic reliability parameters shown in Table 5 are, respectively, fitted as Figures $6,7,8$, and 9 . In Figure 6, we can find that the availability quickly increases at the beginning of the simulation, then slowly changes, and finally reaches almost constant. However, the curve trend of failure rate in Figure 7 just is opposite, which is decreasing rapidly at the beginning of the simulation, then slowly changing, and finally reaching almost constant. 
TABLE 5: Dynamic reliability parameters of servo valve.

\begin{tabular}{lcccc}
\hline Simulation time h & $A$ & $\begin{array}{c}\lambda \\
10^{-5}\end{array}$ & $\begin{array}{c}\text { MTTR } \\
\mathrm{h}\end{array}$ & $\begin{array}{c}\text { MTBF } \\
\mathrm{h}\end{array}$ \\
\hline 1000 & 0.943 & 1.945 & 1894 & 49304 \\
10000 & 0.957 & 1.763 & 1280 & 54407 \\
100000 & 0.961 & 1.585 & 1172 & 60527 \\
1000000 & 0.962 & 1.530 & 1149 & 62689 \\
10000000 & 0.963 & 1.475 & 1124 & 65035 \\
\hline
\end{tabular}

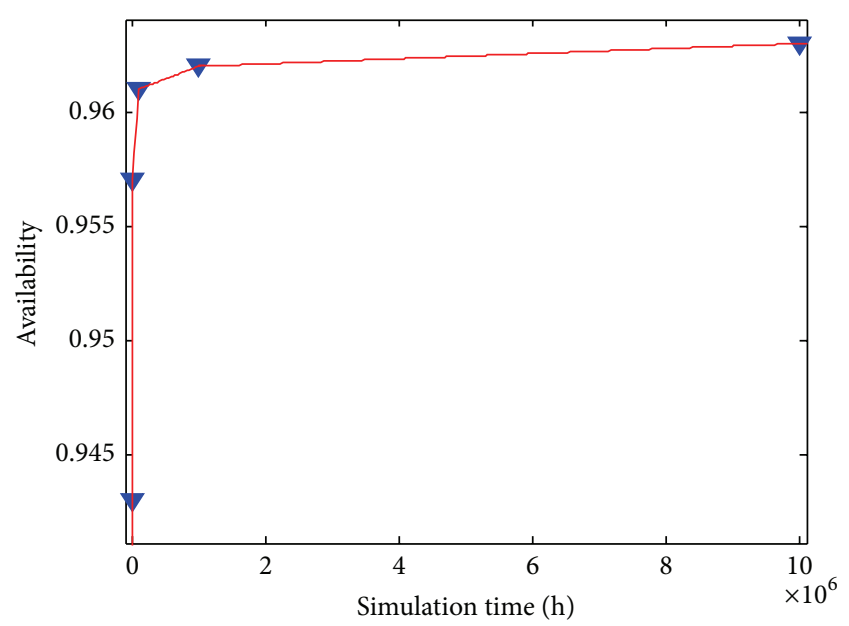

FIGURE 6: The relationship curve between availability and simulation time.

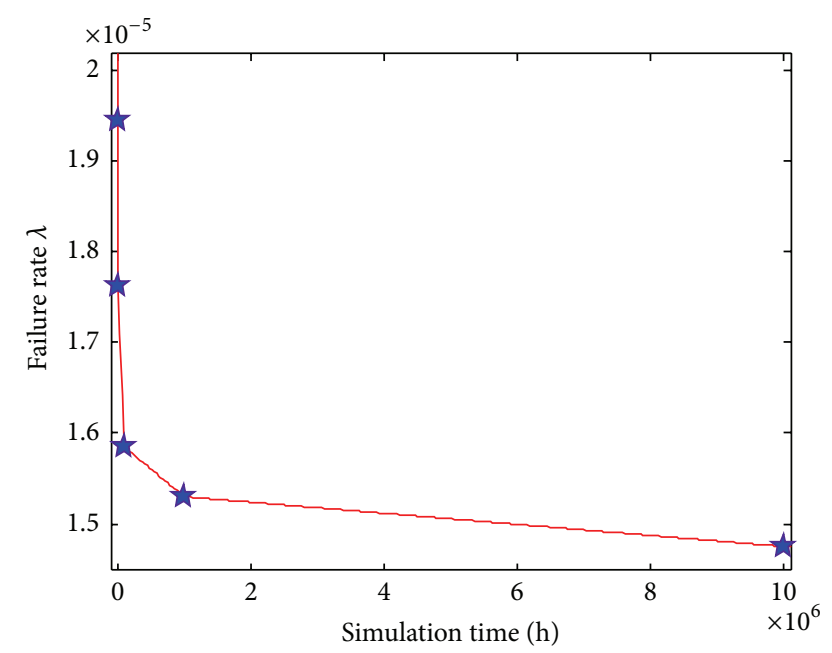

FIGURE 7: The relationship curve between failure rate and simulation time.

The curve trend of MTTR and MTBF is shown in Figures 8 and 9, which are, respectively, the same as Figures 7 and 6.

\section{Results and Discussion}

The systems of military equipment are increasingly large and complex. When assessing their reliability, it is either to need

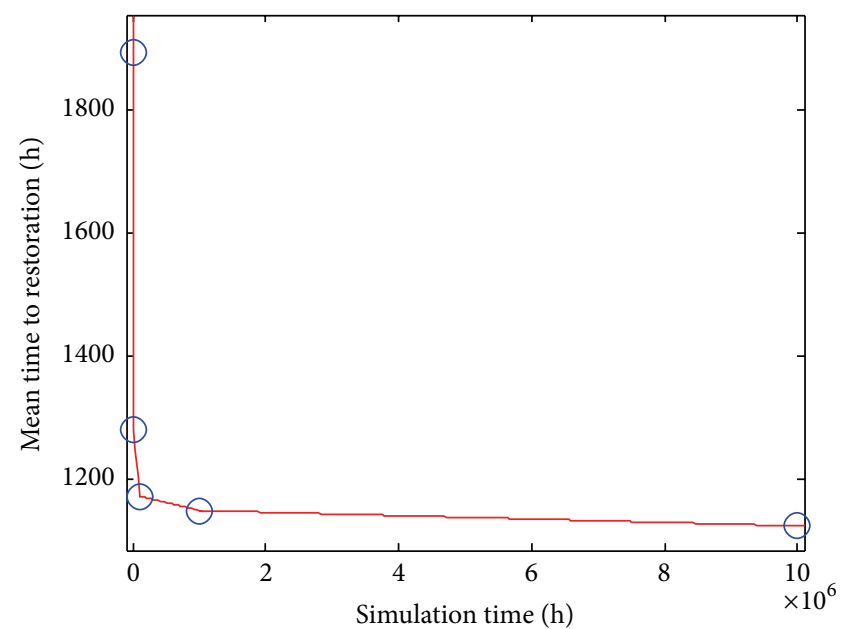

FIGURE 8: The relationship curve between mean time to restoration and simulation time.

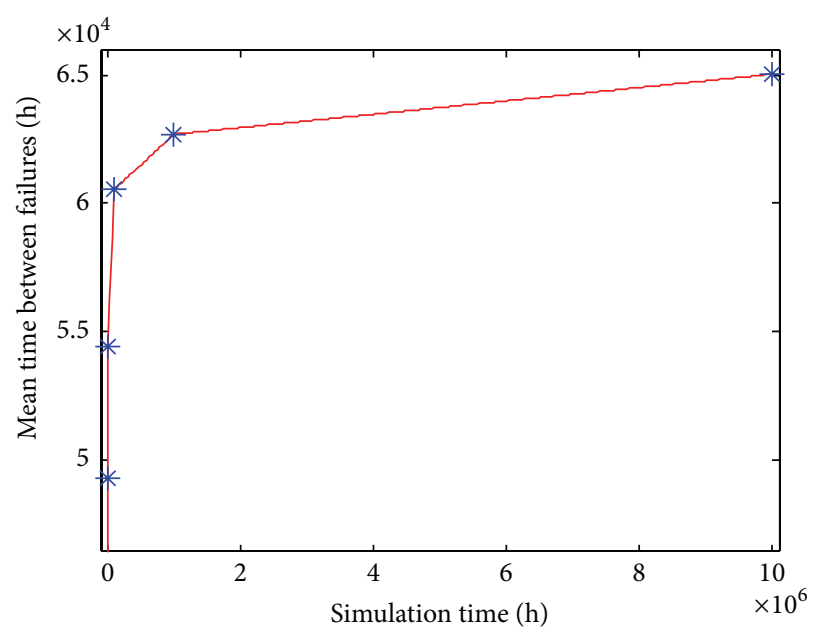

Figure 9: The relationship curve between mean time between failures and simulation time.

solving complex theoretical formulas or to require ignoring too many factors to lead unrealistic in traditional methods. On the basis of GSPN, this paper presents a new method for assessing dynamic reliability considering failure's random and repair, which are concise and realistic for dynamic reliability's quantitative calculation of large and complex systems.

As we all know, repair rate is a double-edged sword. System reliability is not effectively satisfied if repair rate is too small. On the contrary, the maintenance cost increases. The general practice is that the repair rate is determined on the base of reliability requirement's meeting of specific system and considering a certain safety margin. According to the proposed method, the availability of servo valve decreases linearly with the increasing of initial repair rate's multiple after working to required time. The suitable repair rate set of servo valve is obtained by simulation experiments.

The result of simulation experiments is fitted using MATLAB. From Figure 7, we can clearly find the failure rate 
curve is the same as the first half of bathtub curve because of assuming all subsystems are repaired as new, which indicates that the repair of system can effectively extend length of the failure rate curve's stationary phase. From availability curve in Figure 6, it is found that the availability of servo valve increases rapidly at the beginning and finally reaches constant. In Figures 8 and 9, the MTTR and MTBF curves, respectively, have the general trend as failure rate curve and availability curve, which strongly indicates the ability to work properly improves and the ability of recovering from failure gets better. It is clearly concluded from the above dynamic reliability curves of servo valve that the determined repair rate plays a very good role in promoting reliability parameters of servo valve at the beginning; however, this promoting effect becomes weak with the extension of simulation time.

\section{Conclusion}

Facing the dynamic reliability problem of increasingly large and complex military equipment, this paper brings in GSPN theory and presents a novel method assessing dynamic reliability, which is validated using Markov method. The repair rate is optimized according to reliability centered maintenance theory. The optimized repair rate is determined on the basis of required reliability when reaching a certain working time. The dynamic reliability parameters considering failure's randomness and repair are in line with actual working conditions. From the calculational results, we can clearly conclude that the reliability parameters of servo valve get better because of failure's repair. The GSPN method and the calculational results in this study provide an effective way for failure's qualitative forecast and dynamic reliability's quantitative calculation of similar complex systems.

\section{Conflict of Interests}

The authors declare that there is no conflict of interests regarding the publication of this paper.

\section{References}

[1] A. M. Deng, Research on Reliability Technology of HighReliability and Long-Lifetime Products, National University of Defense Technology, 2006.

[2] Y. J. Yuan, The study on the modern production of military products by Q [M.S. thesis], Tianjin University, Tianjin, China, 2013.

[3] C. Su, G. Shen, and Y. Q. Xu, "Modeling theory and it's application for dynamic reliability of manufacturing system," Machine Design and Research, vol. 22, no. 5, pp. 18-22, 2006.

[4] N. Siu, "Risk assessment for dynamic-systems-an overview," Reliability Engineering and System Safety, vol. 43, no. 1, pp. 4373, 1994.

[5] E. Chatelet, E. Zio, and S. Pasquet, "The use of neural networks in the reliability analysis of dynamic systems:an overview," in Proceedings of the 5th International Workshop on Dynamic Reliability: Future Directions, Greenbelt, Md, USA, September 1998.

[6] M. S. Rao and V. N. Naikan, "Reliability analysis of repairable systems using system dynamics modeling and simulation,"
Journal of Industrial Engineering International, vol. 10, no. 3, article 69, 2014.

[7] T. Matsuoka and M. Kobayashi, "New reliability analysis methodology," Nuclear Science and Engineering, vol. 98, pp. 64$78,1988$.

[8] C. Y. Huang and Y. R. Chang, "An improved decomposition scheme for assessing the reliability of embedded systems by using dynamic fault trees," Reliability Engineering and System Safety, vol. 92, no. 10, pp. 1403-1412, 2007.

[9] V. Volovoi, "Modeling of system reliability Petri nets with aging tokens," Reliability Engineering and System Safety, vol. 84, no. 2, pp. 149-161, 2004.

[10] A. Ejlali and S. G. Miremadi, "FPGA-based Monte Carlo simulation for fault tree analysis," Microelectronics Reliability, vol. 44, no. 6, pp. 1017-1028, 2004.

[11] C. Gianfranco, G. Reinhard, L. Christoph et al., "Acharacterization of the stochastic process undering a stochastic petri net," IEEE Transactions on Software Engineering, vol. 20, no. 7, pp. 506-515, 1994.

[12] G. Zhou, Y. Qian, and T. Q. Lv, "Reliability analysis of jettube hydraulic servo valve based on FMECA method," Ship Engineering, vol. 36, no. 4, pp. 57-60, 2014.

[13] C. G. Dai, X. H. Wang, X. Zhang, and L. Z. Wang, "Fuzzy comprehensive evaluation in FMECA of electro-hydraulic servo valve," Journal of Beijing University of Aeronautics and Astronautics, vol. 37, no. 12, pp. 1575-1578, 2011.

[14] T. Q. Lyu, G. Zhou, and Y. Qian, "Reliability digital simulation of jet-tube hydraulic servo valve based on fault tree method," Ship Standardization Engineer, vol. 47, no. 4, pp. 52-55, 2014.

[15] G. Balbo, "Introduction to stochastic petri nets," in Lectures on Formal Methods and Performance Analysis, vol. 2090 of Lecture Notes in Computer Science, pp. 84-155, Springer, Berlin, Germany, 2001.

[16] C. Su, "Simulation research on system dynamic reliability based on general stochastic petri net," China Mechanical Engineering, vol. 19, no. 1, pp. 1-5, 2008. 

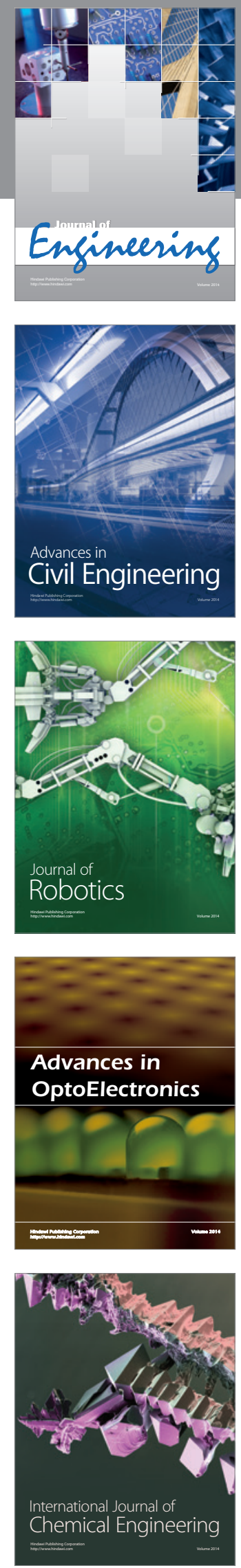

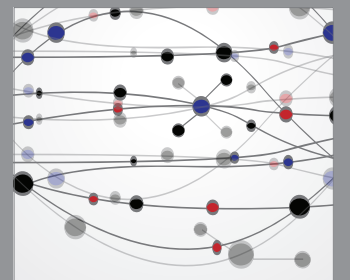

The Scientific World Journal
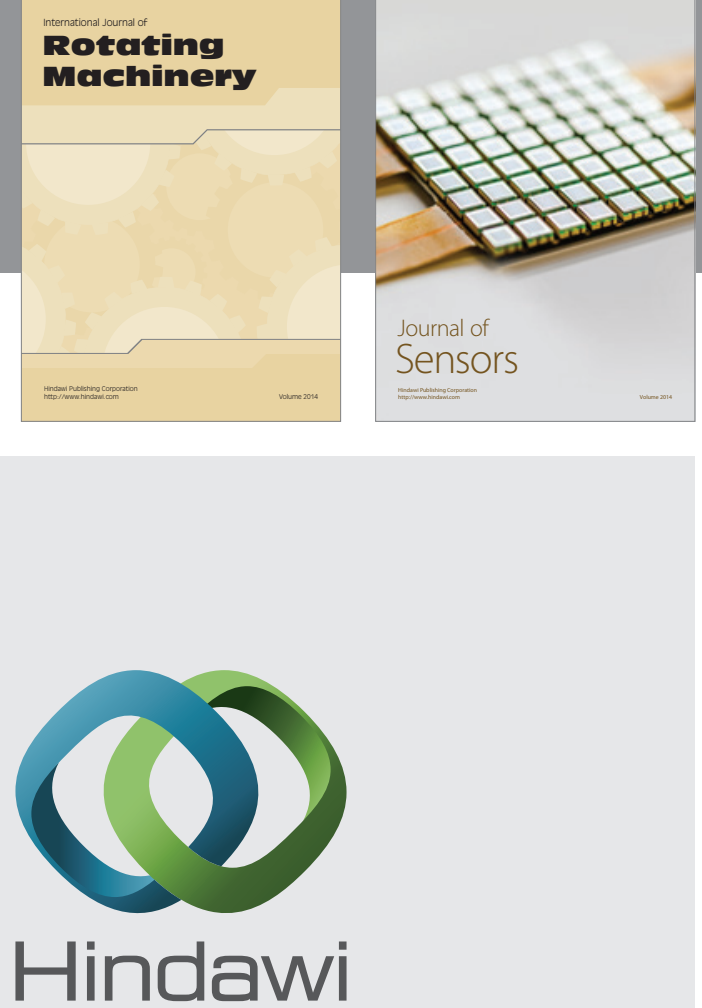

Submit your manuscripts at http://www.hindawi.com
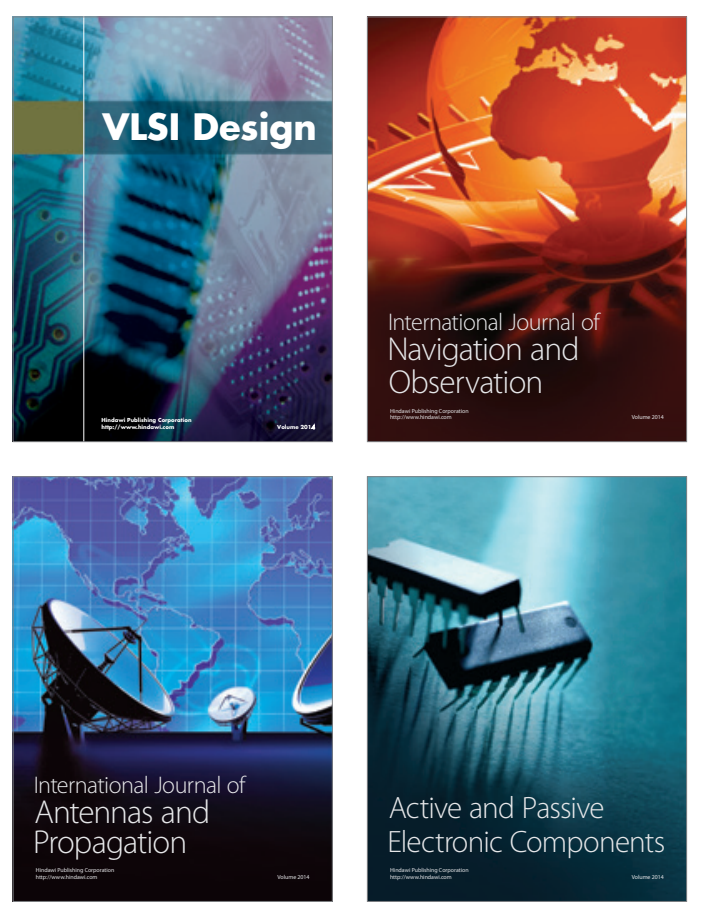
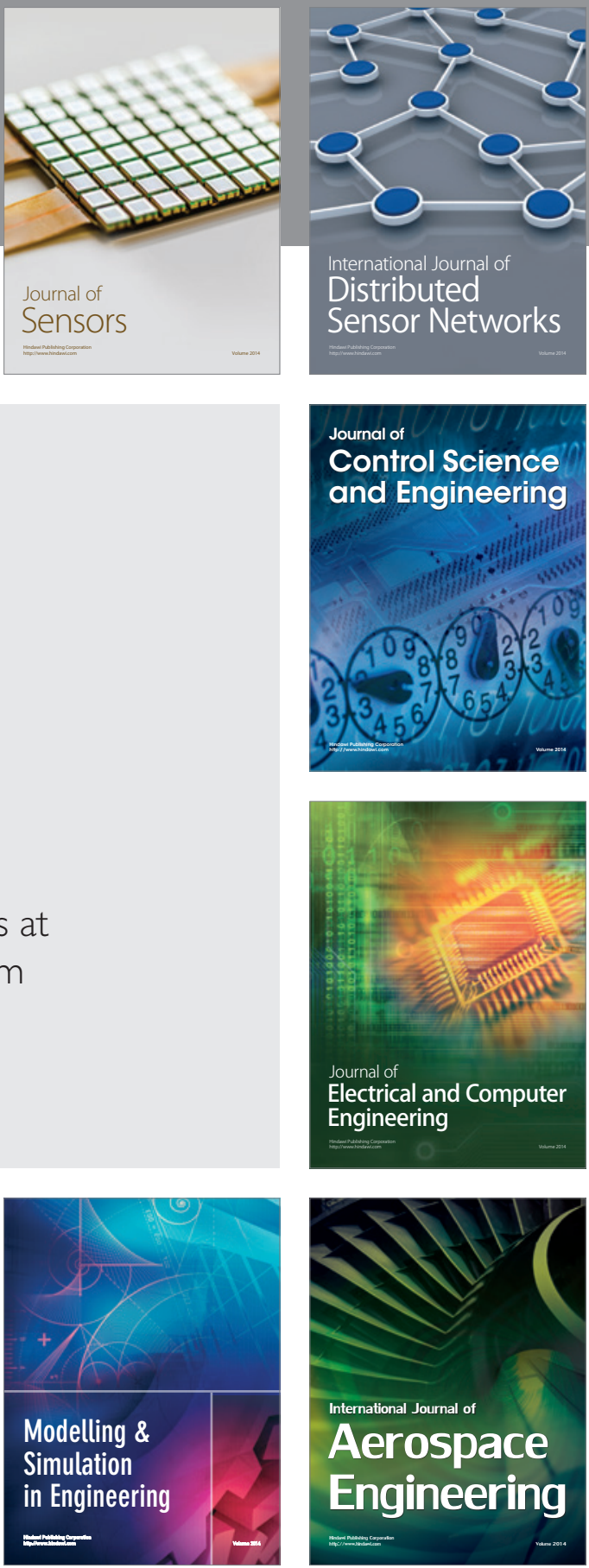

Journal of

Control Science

and Engineering
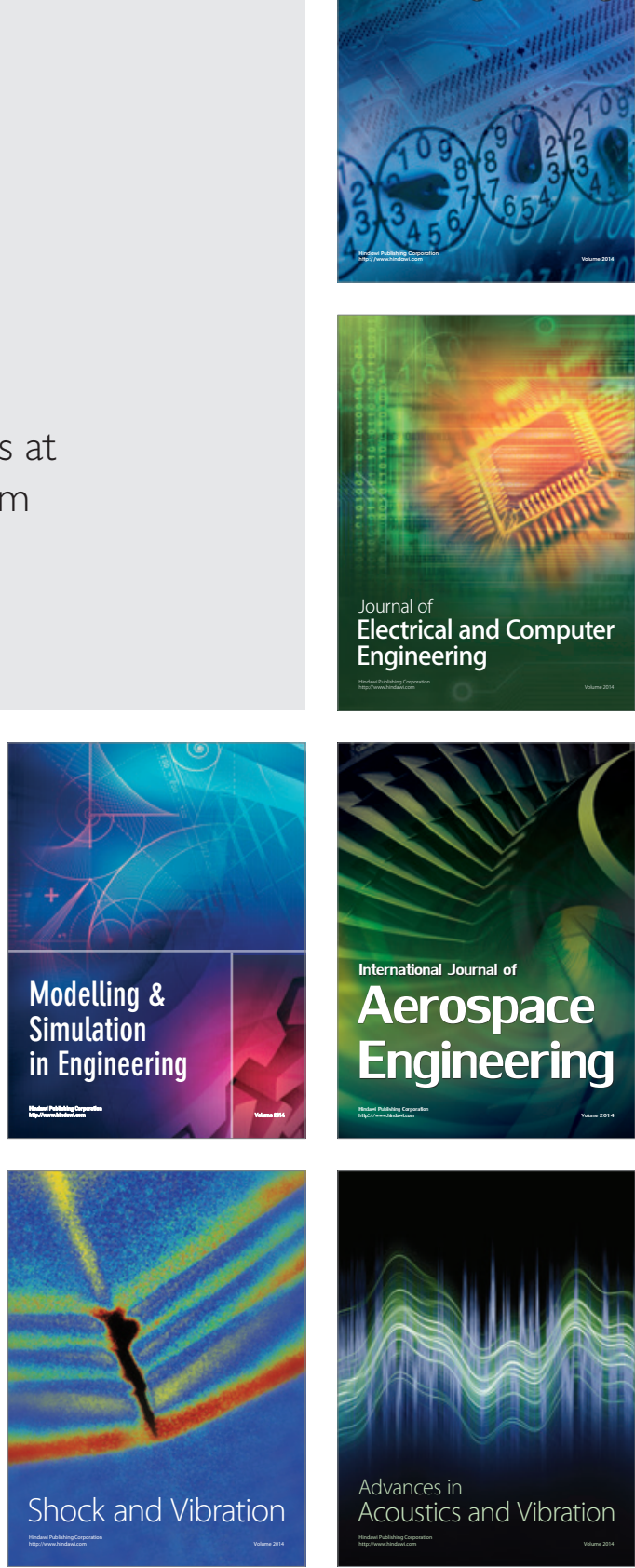